\title{
CARACTERÍSTICAS DO CUIDADO EM SAÚDE MENTAL EM UM CAPS NA PERSPECTIVA DOS PROFISSIONAIS
}

\author{
CHARACTERISTICS OF MENTAL HEALTH CARE IN A CAPS FROM THE PERSPECTIVE \\ OF THE PROFESSIONALS
}

\author{
Fernanda Barreto Mielke ${ }^{1}$ \\ Luciane Prado Kantorski ${ }^{2}$ \\ Agnes Olschowsky ${ }^{3}$ \\ Vanda Maria da Rosa Jardim ${ }^{4}$
}

Resumo Neste estudo qualitativo, subprojeto da pesquisa "Avaliação dos CAPSs da região sul do Brasil", temos o objetivo de conhecer as características do cuidado em saúde mental oferecido por um centro de atenção psicossocial (CAPS) na perspectiva de seus profissionais. A coleta de dados foi realizada por meio de entrevistas individuais. Utilizou-se a análise temática, emergindo dos dados três temáticas. Neste artigo, destacamos a temática intitulada 'caracterização do cuidado em saúde mental prestado pelos profissionais do CAPS'. As características do cuidado em saúde mental advindas das entrevistas foram autonomia do usuário, que, juntamente com a questão da alta do serviço, deve ser mais desenvolvida; atendimento centrado especificamente na doença como herança do tratamento hospitalar; e preocupação da equipe com a implementação de práticas psicossociais inclusivas e cidadãs.

Palavras-chave saúde mental; assistência à saúde; serviços de saúde.

Abstract In this qualitative study, a sub-project of the "Evaluation of CAPSs in Southern Brazil" research project, the goal is to get to know the characteristics of mental health care offered by a psychosocial care center (CAPS) in view of its professionals. Data collection was conducted through individual interviews. Thematic analysis was used, and three themes stemmed from the data. In this article, we highlight the theme titled 'characterization of the mental health care provided by the CAPS professionals.' The characteristics of mental health care noticed in the interviews were user autonomy, which, together with the issue of discharge from the service, should be further developed; care focused specifically on the disease as a legacy of hospital care; and the team's concern with the implementation of inclusive psychosocial practices.

Keywords mental health; health care; health services. 


\section{Introdução}

A reforma psiquiátrica brasileira, caracterizada como um movimento político, social e ideológico, compreende um conjunto de transformações de práticas, saberes, valores culturais e sociais, e está pautada na desinstitucionalização e na reabilitação psicossocial. Isso repercute na transformação do modelo assistencial em saúde mental, anteriormente pautado na hospitalização, na medicalização e na doença mental (Brasil, 2005; Costa-Rosa, 2000).

Destacamos a reabilitação psicossocial, entendida como um conjunto de ações que visa a aumentar as habilidades da pessoa, diminuindo o dano causado pelo transtorno mental, e que envolve todos aqueles que fazem parte do processo saúde-doença, ou seja, usuários, familiares, profissionais e comunidade em geral (Lussi, Pereira e Pereira Júnior, 2006). O propósito da reabilitação é valorizar, utilizando diferentes recursos e serviços, as potencialidades e capacidades do indivíduo que sofre psiquicamente.

A reabilitação psicossocial é um processo que deve contemplar os três vértices da vida humana: casa, trabalho e lazer. É uma ação ampliada que se configura como um conjunto de estratégias direcionadas a aumentar a capacidade de trocas e a valorizar a subjetividade, proporcionando contratualidade e solidariedade (Saraceno, 2001; Pereira, 2007). E para que esse processo seja efetivo, é imprescindível que o indivíduo em sofrimento psíquico seja reinserido na vida em sociedade. A reinserção consiste na retomada da autonomia e da cidadania, quando a pessoa conquista a sua liberdade e exercita a subjetividade, circulando nos espaços da cidade e promovendo novas relações sociais.

Pautada no modo psicossocial, constituído a partir da crítica ao modo manicomial e com base no exercício prático de novas formas de cuidar em saúde mental que valorizam e singularizam a subjetividade da pessoa em sofrimento psíquico (Costa-Rosa, 2000), a reabilitação psicossocial utiliza-se dos dispositivos instalados na comunidade, o território onde a vida social acontece.

O indivíduo é compreendido como protagonista do seu tratamento e inserido em um grupo familiar - grupo que também é atendido pelos serviços de saúde (Costa-Rosa, 2000). Assim, as atividades dos centros de atenção psicossocial (CAPSs) incluem o cuidado à família e estão comprometidas com a inserção social do usuário. Para o desenvolvimento das práticas, a reabilitação se utiliza de aspectos relacionais e dialógicos - entre outros, acolhimento, escuta e vínculo. Nesses aspectos estão pautadas as ações desenvolvidas nos serviços de saúde mental.

A Rede de Atenção Integral em Saúde Mental, cuja ampla estrutura destina-se ao atendimento de necessidades diversas, abrange serviços de todos os níveis de atenção, desde a atenção básica até os serviços especia- 
lizados: centros de atenção psicossocial, serviços residenciais terapêuticos, ambulatórios de saúde mental, hospitais-dia, leitos/unidades em hospitais gerais, serviços de urgência/emergência psiquiátrica, geração de renda e trabalho, centros de convivência, pensão protegida, entre outros (Brasil, 2001).

Dentro dessa rede, particularizamos os CAPSs: serviços de atenção diária que se responsabilizam pelo cuidado de pessoas com transtornos mentais severos e persistentes, trabalhando com a lógica do território. $\mathrm{O}$ atendimento está baseado no acolhimento, no vínculo e na responsabilização de cada membro da equipe multiprofissional (Brasil, 2004). Tendo em vista as suas características de inclusão e a participação do usuário, esses serviços têm sido considerados fundamentais para a continuidade do processo de reabilitação psicossocial.

A reabilitação psicossocial define-se como um saber-fazer que considera o transtorno psíquico como um dado a mais na história de vida do indivíduo, que é visto como uma pessoa que vive em um território, que mantém relações sociais e afetivas e que faz parte de determinada família, e que, além disso, apresenta um transtorno mental que repercute de diferentes maneiras em seu cotidiano (Kantorski et al., 2006). O foco do trabalho desenvolvido nos serviços substitutivos, e especificamente nos CAPSs, não é a doença, mas sim, prioritariamente, o usuário e seu entorno social.

Os CAPSs são definidos e regulamentados pela portaria ministerial n. 336, de 19 de fevereiro de 2002 (Brasil, 2002), e devem oferecer atendimento individual, grupal, oficinas terapêuticas, visitas domiciliares, atendimento à família e atividades comunitárias. De acordo com a população dos municípios, os CAPSs são classificados como I, II ou III.

Para avaliar a eficiência e a eficácia dos CAPSs, é necessário conhecer as práticas desenvolvidas pelos profissionais desse serviço e compará-las aos preceitos teóricos que as permeiam e que se baseiam no modo psicossocial para o cuidado em saúde mental. No presente artigo, temos o objetivo de conhecer as características que compõem o cuidado em saúde mental oferecido por um CAPS de tipo II, características que foram elencadas pelos profissionais do referido serviço.

\section{Metodologia}

O presente estudo é um subprojeto da pesquisa "Avaliação dos centros de atenção psicossocial da região sul do Brasil - CAPSUL", aprovada pelo Comitê de Ética em Pesquisa da Faculdade de Medicina da Universidade Federal de Pelotas (UFPel), ofício n. 068/06. A pesquisa foi realizada pela Faculdade de Enfermagem da Universidade Federal de Pelotas, em parceria com a Escola de Enfermagem da Universidade Federal do Rio Grande do Sul 
e com o Curso de Enfermagem da Universidade Estadual do Oeste do Paraná (Campus Cascavel). O estudo compreendeu duas etapas de coleta de dados: quantitativa e qualitativa. Os dados foram coletados em municípios dos estados do Rio Grande do Sul, Santa Catarina e Paraná.

O subprojeto também foi desenvolvido em duas etapas. A primeira, de caráter quantitativo, foi desenvolvida com base nos dados quantitativos coletados no estudo-piloto da pesquisa CAPSUL, realizada em um CAPS do estado do Rio Grande do Sul. A análise descritiva desses dados permitiu a organização e a formulação do instrumento de pesquisa da segunda etapa, essa já de cunho qualitativo, realizada no mesmo serviço de saúde mental onde foi feita a primeira etapa. Neste artigo, apresentamos dados parciais da etapa qualitativa, caracterizada como descritiva e exploratória.

A pesquisa qualitativa é fundamental para atingir o objetivo de conhecer as características do cuidado oferecido aos indivíduos em sofrimento psíquico em um serviço substitutivo conforme o entendimento dos seus profissionais, pois permite desvelar processos sociais pouco conhecidos e construir novas abordagens, conceitos e categorias durante a investigação, sendo aplicável ao estudo das relações, percepções e opiniões da interpretação humana (Minayo, 2007).

Os sujeitos do estudo foram 18 profissionais de um universo de 22 - dos demais, três estavam em período de férias na época da coleta dos dados e um não aceitou participar. Os profissionais que concordaram em participar do estudo assinaram o Termo de Consentimento Livre e Esclarecido, que assegura o anonimato dos participantes e autoriza a utilização e a publicação dos dados obtidos. Os aspectos éticos foram devidamente assegurados, em concordância com a resolução n. 196/1996 do Conselho Nacional de Saúde do Ministério da Saúde (Brasil, 1996).

A coleta de dados qualitativos ocorreu no mês de dezembro de 2006. Ela se deu por meio de entrevistas semiestruturadas, que foram gravadas e transcritas na íntegra, partindo da seguinte questão norteadora: 'Como o(a) sr.(a) caracteriza o cuidado em saúde mental prestado ao usuário pelos profissionais do CAPS?'. Os profissionais foram identificados pela letra T, de trabalhador, seguida pelo número da ordem de realização da entrevista.

Para a análise dos dados, utilizamos a análise temática, operacionalizada por meio de três etapas: pré-análise; exploração do material e tratamento dos resultados obtidos; e interpretação (Minayo, 2007). Ao final desse processo, obtivemos três temáticas, dentre as quais destacaremos a intitulada 'caracterização do cuidado em saúde mental prestado pelos profissionais do CAPS', que será desenvolvida neste artigo.

A temática em destaque aborda questões referentes à autonomia dos usuários, ao atendimento centrado especificamente na doença e à preocupação da equipe com o cuidado aos usuários, aspectos mencionados pelos 
sujeitos do estudo como características do cuidado em saúde mental desenvolvido no serviço estudado.

\section{Resultados e discussão}

O serviço em estudo localiza-se em um município da região sul do Brasil, no estado do Rio Grande do Sul, tendo sido cadastrado em 2002, no Ministério da Saúde, como Centro de Atenção Psicossocial II (CAPS II). Ressalte-se que alguns profissionais da atual equipe realizavam atendimentos em saúde mental em uma unidade básica de saúde desde 1992.

A equipe compreende um total de 22 profissionais de níveis médio e superior, de diversas categorias profissionais: médico, enfermeiro, psicólogo, artista plástico, músico, professor de educação física, cozinheiro, auxiliar de serviços gerais e auxiliar administrativo. O serviço funciona das 8 às 12 horas e das 13 às 17,30 horas.

No serviço estudado, são atendidos cerca de 600 usuários por mês, distribuídos nas modalidades intensivo, semi-intensivo e não intensivo, conforme preconizado pela portaria ministerial n. 336/2002. Nele são desenvolvidas atividades como triagem, atendimento em grupo e individual, oficinas terapêuticas, atendimento à família, assembleia de usuários, visitas domiciliares, atividades físicas e lúdicas e reunião de equipe. O serviço possui coordenação local eleita pelos usuários, sendo que, no período da coleta de dados, a coordenadora era uma psicóloga que ocupava o cargo há um ano e três meses.

A partir da questão norteadora, os sujeitos do estudo indicaram algumas características do cuidado em saúde mental oferecido no CAPS. A primeira característica refere-se à construção da autonomia do usuário, questão fundamental quando perseguimos a proposta da reabilitação psicossocial.

Pautamo-nos num conceito de autonomia que a considera como o momento em que o usuário consegue viver a vida, necessitando cada vez menos da assistência do serviço (Santos et al., 2000). Ou seja, trata-se de incentivar o usuário a ampliar a sua rede social, sem restringi-la exclusivamente aos vínculos estabelecidos dentro do serviço, favorecendo sua inclusão na sociedade e estimulando a não dependência ao serviço.

Os profissionais do CAPS entendem que a construção da autonomia do usuário faz parte do cuidado oferecido nesse serviço. Por ser um dispositivo do modo psicossocial, o CAPS deve atuar na reinserção social do usuário, tendo como base o resgate da autonomia. As atividades desenvolvidas pelos profissionais necessitam ir ao encontro desse pensamento, sendo realizadas prioritariamente na comunidade.

Nesse sentido, o CAPS desenvolve ensaios de grupo de dança e atividades físicas em espaços da comunidade - respectivamente, no centro de 
tradições gaúchas e em uma quadra de futebol do bairro. Essas atividades permitem que os usuários circulem nesses espaços sociais e que sejam vistos pela comunidade como cidadãos. Os profissionais também estimulam a participação dos usuários em festas comunitárias e eventos sociais, dentre os quais se destacam a feira de artesanato da cidade - na qual uma banca de venda de produtos produzidos nos CAPS do município é mantida por usuários e seus familiares - e as festas temáticas - Carnaval, Páscoa e Natal -, que contam com a participação da comunidade e são realizadas em clubes ou salões de festas da cidade.

Ainda assim, a construção da autonomia dos usuários é prejudicada por vezes por atitudes paternalistas e pelo 'excesso de cuidado', que se contrapõem às atividades de reinserção social motivadas pelo CAPS estudado:

(...) a gente, às vezes, cria uma dependência que não deveria, aí tu não tens como desenvolver, a pessoa é o máximo aqui dentro, faz tudo aqui dentro, pinta, faz poema, mas lá na rua ela não é nada (...). “Ah, eu vou ficar no CAPS, porque aqui é a minha casa" - outra coisa que eu não gosto de ouvir (...) tu podes até voltar um dia, mas vem só nos visitar, não ficar aqui, aqui não é lugar. Tem gente aqui que nem deveria estar aqui (...) o X [usuário] era para estar dentro de uma oficina ajudando, sendo auxiliar de marcenaria, trabalhando (...) (T5).

A fala descrita acima demonstra que o serviço tem dificuldade de discutir com os usuários a questão de que o CAPS, respeitada a sua especificidade, é um serviço de saúde como outro qualquer - isto é, o tratamento ali oferecido tem início, meio e fim. $\mathrm{O}$ atendimento ao usuário deve ocorrer de acordo com a sua necessidade e pautando-se na compreensão de que a alta faz parte do tratamento. Não havendo essa compreensão, passa a haver risco de cronificação dos usuários dentro do CAPS. E isso porque, muitas vezes, o serviço não é visto pelos usuários como um local apenas de suporte terapêutico, mas sim como o único espaço de troca social. Isso gera dependência em relação ao serviço e interfere negativamente na construção da autonomia do usuário (Mello e Furegato, 2008).

Os profissionais dos CAPS têm mostrado preocupação com a 'institucionalização velada' do usuário que decorre de sua longa permanência em atendimento nesses locais (Mello e Furegato, 2008). Com isso, estaria ocorrendo um retrocesso ao modo hospitalocêntrico, no qual a alta não é efetivada pela incapacidade de o doente mental, por causa dos longos anos de exclusão, levar uma vida em sociedade.

No CAPS estudado, o usuário tem a liberdade de ir e vir no espaço social, mas sua postura ante a sociedade não é a de um cidadão com autonomia para levar a vida. É importante o entendimento de que a reinserção social não consiste em 'devolver' o usuário à sociedade, e sim que implica a 
invenção de novas possibilidades de inscrição dos sujeitos no corpo social (Amarante, 1996). Além disso, é preciso ter em conta que a alta não significa desassistência; ao contrário, reflete a possibilidade de articulação com os demais serviços da rede de saúde mental que atendam ao usuário em suas necessidades, no momento em que ele deixa de necessitar do atendimento intensificado e especializado do CAPS.

É primordial discutir com o usuário os benefícios e as oportunidades que a alta lhe proporcionará, mostrando a ele que não será um sofrimento deixar de ir ao serviço e sim a conquista de outra fase importante do tratamento proposto no CAPS, que lhe permitirá construir novos vínculos com outros serviços e pessoas, ampliando sua rede social.

Entendemos que práticas voltadas à autonomização do usuário devem ser perseguidas pelos profissionais do CAPS, uma vez que esse recurso de saúde tem condições de contribuir para que o usuário retome a sua vida de maneira mais independente e autônoma. Sabemos que não se trata de tarefa fácil, mas ela não deve ser esquecida no cotidiano do trabalho do CAPS.

Outra característica do cuidado em saúde mental oferecido no CAPS estudado é o fato de o atendimento ao usuário ainda ser voltado para a doença. Consideramos que essa prática é herança do tipo de tratamento que a maioria dos usuários conheceu nos manicômios - e também da formação dos profissionais no modelo biomédico.

A formação dos profissionais de saúde é centrada nos aspectos técnicos e científicos, tendo como referência o diagnóstico, o cuidado, o tratamento, o prognóstico, a etiologia e a profilaxia de doenças e agravos, sem estar integrada às necessidades de saúde das pessoas e populações, à gestão setorial e ao controle social em saúde (Ceccim e Feuerwerker, 2004). A atenção esperada em saúde mental, de acordo com os princípios da reabilitação psicossocial, exige dos profissionais novas habilidades e competências que não são encontradas no cotidiano da formação em saúde, ou que estão inseridas de forma aleatória, pontual e precária nos currículos (Oliveira, 2007).

Da mesma forma, o usuário também tem a doença como aspecto central de sua existência, pois o saber médico psiquiátrico instituído nos manicômios destina sua atenção à doença mental e sua sintomatologia. Os profissionais do CAPS têm buscado incentivar o usuário para que fale de sua saúde, de suas conquistas com relação ao transtorno mental, fazendo-o perceber que o essencial é o ser humano, ele, e não sua doença.

(...) eu penso que o cuidado aqui está um pouco mais direcionado para a resolução de problemas; muito mais para o cuidado com o sintoma em si do que para o cuidado com a vida como um todo (...). Nos grupos que eu atendo, eu procuro direcionar um pouco mais o trabalho para esse lado da própria pessoa cuidar da vida dela como um todo, procurando mostrar que esse cuidado com a vida como 
um todo faz que a pessoa melhore a saúde mental (...), e que isso seja valorizado de uma maneira absoluta (...) (T9).

A consciência da equipe sobre esse aspecto é importante e demonstra a necessidade de mudanças em relação ao enfoque dado nos atendimentos, abrindo espaços e instigando o usuário a falar de suas potencialidades.

Pautada nos aspectos relacionais, a reabilitação psicossocial exige dos profissionais uma nova prática em saúde mental que leve em consideração os diferentes fatores envolvidos na vida humana - político, econômico, social, cultural e familiar, além do biológico -, modifique os conceitos de cura, doença e tratamento, e enfoque o cuidado na subjetividade. O olhar deixa de ser exclusivamente técnico e clínico, o que, na tradição basagliana, seria colocar a doença entre parênteses (Amarante, 1996).

Não podemos negar, porém, a existência da doença concreta nem negligenciá-la, como defendem algumas linhas de pensamento. Negar as dificuldades impostas pela convivência com o sofrimento psíquico não constrói cuidado em saúde mental, pois é a idealização do sofrimento, o que não auxilia verdadeiramente o usuário em sua vida social.

A última característica do cuidado em saúde mental é a preocupação da equipe com os usuários. A equipe do serviço vem trabalhando junta há alguns anos, o que facilita o entendimento entre os profissionais, apesar da diversidade de concepções sobre saúde mental.

O trabalho em equipe na atenção psicossocial permite ao usuário receber assistência de qualidade, pois o objeto de cuidado não se limita à doença mental, mas envolve relações sociais, aspectos emocionais, afetivos e biológicos (Tavares, 2005).

As práticas terapêuticas que têm como objetivo a reabilitação da pessoa que sofre psiquicamente exigem um trabalho coletivo no qual estão previstas intervenções multidisciplinares que incluem a participação do usuário e da família (Kantorski, Mielke e Teixeira Júnior, 2008; Brasil, Jorge e Costa, 2008) e, por isso, implicam criatividade e a interação de várias áreas.

O envolvimento da equipe com as atividades do CAPS faz extrapolar muitas vezes - quando, por exemplo, levam para casa tarefas que não foi possível concluir no serviço - a carga horária desses profissionais:

(...) eu acabei fazendo o restante das bombachas, aprendi a fazer bombacha, ia até as três horas da madrugada fazendo, (...) a maior realização, porque, na realidade, todas as horas que eu doei a mais, indo até a madrugada costurando, até envolvendo a minha família - porque [até] três horas da madrugada (...) a família ficava acordada, por causa do barulho da máquina, mas poder ver que eles estão arrumados e felizes compensa o trabalho que a gente faz de coração (...), que não é recompensado por dinheiro, mas sim pela satisfação de ver que aquele objetivo foi concretizado (T13). 
A fala de T13 remete ao compromisso que os profissionais do serviço têm com a proposta de atenção psicossocial e, especialmente, com os usuários, pois de nada adiantaria ter todo o aparato clínico se não houvesse a comunicação, a preocupação e o afeto em cada ação dos profissionais. A reabilitação psicossocial implica a responsabilização dos profissionais nas atividades de reinserção social dos usuários, sendo a equipe um apoio na reconstrução dos laços sociais fragilizados (Schneider, Camatta e Nasi, 2007).

Compreendemos que é fundamental para a efetividade do trabalho em saúde mental, na perspectiva da reabilitação psicossocial, que o profissional acredite na sua prática terapêutica, desenvolvendo-a com habilidade, responsabilidade e satisfação para a promoção do cuidado.

Entendemos que as características do CAPS elencadas pelos profissionais acenam para a proposta de reabilitação psicossocial, destacando, também, a vinculação e a afetividade entre profissionais e usuários. Apesar de a questão da alta e do atendimento estar centrada na doença - o que vemos como obstáculos a serem transpostos -, percebemos que o serviço tem buscado incentivar os usuários a ampliar sua rede social, o que pode facilitar a construção da autonomia, da cidadania e da reinserção social.

\section{Considerações finais}

A realização deste estudo possibilitou que se conhecesse a preocupação dos profissionais do CAPS em relação à proposta de reabilitação psicossocial. É importante destacar que este estudo foi realizado no ano de 2006. Devem ser levados em consideração, assim, os aspectos que compunham o cenário do local do estudo na época da coleta de dados. É possível que já tenham ocorrido mudanças em nível local, e até mesmo nacional, que levaram a modificação de alguns aspectos aqui destacados.

A equipe do CAPS estudado tem desenvolvido atividades que visam à reabilitação psicossocial do usuário para a retomada de sua vida na sociedade, como oficinas em espaços da comunidade, mediante parcerias e a participação em eventos do município, entre outros.

Entendemos que a questão da alta e a centralidade ocupada pela doença nos atendimentos são obstáculos a serem transpostos pelo serviço. Podemos dizer que essas questões são próprias do processo de reforma, ou seja, nessa construção há diferentes concepções e atores que, por meio do enfrentamento e da troca de ideias, implantam mudanças na atenção psicossocial. Destacamos a importância do reconhecimento desses aspectos por parte dos profissionais do CAPS estudado, que têm refletido e buscado transformar as condutas estabelecidas, favorecendo a reabilitação psicossocial do usuário. 
O fato de a equipe estar valorizando uma fala dos usuários com outro conteúdo que não seja a doença em si mesma já demonstra uma mudança em relação ao modelo biomédico, visando à superação dele, que, pelo que vimos, não atende todas as necessidades dos sujeitos em sofrimento psíquico.

A preocupação dos profissionais ante a proposta de reabilitação psicossocial aponta para o compromisso que assumem, cotidianamente, com a transformação do modelo assistencial em saúde mental, por acreditarem em práticas terapêuticas inclusivas e reabilitadoras, centrando seu cuidado na existência do usuário com sua história de vida.

O trabalho em saúde mental na concepção da reabilitação psicossocial é coletivo e demanda novas habilidades de todos os atores envolvidos: profissionais, usuários, familiares e comunidade. As conquistas do movimento de reforma psiquiátrica se devem a esses atores, que acreditam e praticam uma forma de cuidado pautada no respeito ao outro e na valorização do ser humano.

\section{Nota do Editor}

Este artigo é parte da monografia "O cuidado em saúde mental no entendimento dos profissionais de um CAPS", requisito para conclusão do curso de graduação em Enfermagem, na Faculdade de Enfermagem e Obstetrícia da Universidade Federal de Pelotas (UFPel), de autoria de Fernanda Barreto Mielke. Contou com apoio do Conselho Nacional de Desenvolvimento Científico e Tecnológico (CNPq). As autoras declararam que trabalharam em conjunto em todas as etapas de produção do artigo.

\section{Notas}

1 Enfermeira assistencial da Secretaria Estadual de Saúde do Rio Grande do Sul, Porto Alegre, Rio Grande do Sul, Brasil. Doutoranda do Programa de Pós-graduação da Escola de Enfermagem da Universidade Federal do Rio Grande do Sul (UFRGS). <fbmielke@gmail.com> Correspondência: Rua Coronel Fernando Machado, 851, apt. 504, CEP 90010-321, Centro, Porto Alegre, Rio Grande do Sul, Brasil. 
2 Enfermeira e professora adjunta da Faculdade de Enfermagem e Obstetrícia da Universidade Federal de Pelotas (UFPel), Pelotas, Rio Grande do Sul, Brasil. Doutora em Enfermagem pela Escola de Enfermagem de Ribeirão Preto, da Universidade de São Paulo (EERP/USP). <kantorski@uol.com.br>

3 Enfermeira e professora adjunta da Escola de Enfermagem da Universidade Federal do Rio Grande do Sul (UFRGS), Porto Alegre, Rio Grande do Sul, Brasil. Doutora em Enfermagem Psiquiátrica pela Escola de Enfermagem da Universidade de São Paulo (EE/USP). <agnes@enf.ufrgs.br>

4 Enfermeira e professora adjunta da Faculdade de Enfermagem e Obstetrícia da Universidade Federal de Pelotas (UFPel), Pelotas, Rio Grande do Sul, Brasil. Doutora em Enfermagem pela Universidade Federal de Santa Catarina (UFSC). <phein@uol.com.br>

\section{Referências}

AMARANTE, Paulo. O homem e a serpente: outras histórias para a loucura e a psiquiatria. Rio de Janeiro: Editora Fiocruz, 1996.

BRASIL, Eysler Gonçalves Maia; JORGE, Maria Salete Bessa; COSTA, Edmara Chaves. Concepções de usuários e trabalhadores de um CAPS da SER-IV, de Fortaleza-CE, acerca do cuidado em saúde mental. Ciência, Cuidado e Saúde, Maringá, v. 7, n. 3, p. 333-338, 2008.

BRASIL. Ministério da Saúde. Conselho Nacional de Saúde. Resolução n. 196, de 10 de outubro de 1996. Aprova as diretrizes e normas regulamentadoras de pesquisas envolvendo seres humanos. Brasília, DF: MS, 1996.

. Ministério da Saúde. Guia de saúde mental. Porto Alegre: Governo do Estado do Rio Grande do Sul, 2001.

. Ministério da Saúde. Portaria GM n. 336, de 19 de fevereiro de 2002. Define e estabelece diretrizes para o funcionamento dos Centros de Atenção Psicossocial. Brasília, DF: MS, 2002.
Ministério da Saúde. Saúde mental no SUS: os centros de atenção psicossocial. Brasília, DF: MS, 2004.

Ministério da Saúde. Secretaria de Atenção à Saúde. Coordenação Geral de Saúde Mental. Reforma psiquiátrica e políticas de saúde mental no Brasil. Brasília, DF: MS, 2005.

CECCIM, Ricardo Burg; FEUERWERKER, Laura C. M. O quadrilátero da formação para a área da saúde: ensino, gestão, atenção e controle social. Physis: Revista Saúde Coletiva, Rio de Janeiro, v. 14, n. 1, p. 41-65, 2004.

COSTA-ROSA, Abílio. O modo psicossocial: um paradigma das práticas substitutivas ao modo asilar. In: AMARANTE, Paulo (org.). Ensaios: subjetividade, saúde mental, sociedade. Rio de Janeiro: Editora Fiocruz, 2000. p. 141-168.

KANTORSKI, Luciane Prado et al. O cuidado em saúde mental: um olhar a partir de 
documentos e da observação participante. Revista de Enfermagem Uerj, Rio de Janeiro, v. 14, n. 3, p. 366-371, 2006.

KANTORSKI, Luciane Prado; MIELKE, Fernanda Barreto; TEIXEIRA JÚNIOR, Sidnei. O trabalho do enfermeiro nos centros de atenção psicossocial. Trabalho, Educação e Saúde, Rio de Janeiro, v. 6, n. 1, p. 87-105, 2008.

LUSSI, Isabela Aparecida de Oliveira; PEREIRA, Maria Alice Ornellas; PEREIRA JÚNIOR, Alfredo. A proposta de reabilitação psicossocial de Saraceno: um modelo de auto-organização? Revista Latino-Americana de Enfermagem, Ribeirão Preto, v. 13, n. 3, p. 448-456, 2006.

MELlO, Rosâne; FUREGATO, Antonia Regina Ferreira. Representações de usuários, familiares e profissionais acerca de um centro de atenção psicossocial. Revista de Enfermagem da Escola Anna Nery, Rio de Janeiro, v. 12, n. 3, p. 457-464, 2008.

MINAYO, Maria Cecília de Souza. O desafio do conhecimento: pesquisa qualitativa em saúde. 10. ed. São Paulo: Hucitec, 2007.

OLIVEIRA, Alice Guimarães Bottaro. Trabalho e cuidado no contexto da atenção psicossocial: algumas reflexões. Revista de Enfermagem da Escola Anna Nery, Rio de Janeiro, v. 10, n. 4, p. 694-702, 2007.
PEREIRA, Maria Alice Ornellas. A reabilitação psicossocial no atendimento em saúde mental: estratégias em construção. Revista Latino-Americana de Enfermagem, Ribeirão Preto, v. 15, n. 4, p. 658-664, 2007.

SANTOS, Núbia Schaper et al. A autonomia do sujeito psicótico no contexto da reforma psiquiátrica brasileira. Psicologia: Ciência e Profissão, Brasília, v. 20, n. 4, p. 46-53, 2000.

SARACENO, Benedetto. A reabilitação como cidadania. In: Libertando identidades: da reabilitação psicossocial à cidadania possível. 2. ed. Rio de Janeiro: TeCorá, 2001. p. 111-142.

SCHNEIDER, Jacó Fernando; CAMATTA, Marcio Wagner; NASI, Cíntia. O trabalho em um centro de atenção psicossocial: uma análise em Alfred Schütz. Revista Gaúcha de Enfermagem, Porto Alegre, v. 28, n. 4, p. 520-526, 2007.

TAVARES, Cláudia Mara de Melo. A interdisciplinaridade como requisito para a formação da enfermeira psiquiátrica na perspectiva da atenção psicossocial. Revista Texto Contexto - Enfermagem, Florianópolis, v. 14, n. 3, p. 403-410, 2005.

Recebido em 18/11/2010

Aprovado em 22/12/2010 